\title{
Population genetics of Bulgarian brown hares Lepus europaeus: allozymic diversity at zoogeographical crossroads
}

\author{
Franz SUCHENTRUNK, Christo MICHAILOV, Georgi MARKOV \\ and Anita HAIDEN
}

Suchentrunk F., Michailov C., Markov G. and Haiden A. 2000. Population genetics of Bulgarian brown hares Lepus europaeus: allozymic diversity at zoogeographical crossroads. Acta Theriologica 45: 1-12.

Allozymic variability of 157 brown hares Lepus europaeus (Pallas, 1778) from eight sampling localities in Bulgaria was studied by horizontal starch gel electrophoresis of 50 putative structural gene loci. The present data were compared to an adjusted data set of brown hares from central Europe (Austria), published earlier, in order to test the hypothesis of increased genetic diversity in brown hares from the southeastern European zoogeographical crossroads region, a possible refuge for brown hares during the last glaciation period. No new polymorphic locus was detected, and only one new allele occurred at a low frequency in the Bulgarian hares. Nevertheless, two estimators of genetic diversity (average expected heterozygosity, Shannon-Weaver information index) where slightly higher in Bulgarian hares than in central European hares. In contrast, some alleles found earlier in various parts of central Europe did not occur in the Bulgarian samples. The latter finding suggests that gene pools of central European brown hares might have received gene flow from other parts of Europe during their postglacial (re-)colonization process. This accords to an alternative hypothesis of postglacial colonization of central Europe from both southeastern and southwestern refuges.

Research Institute of Wildlife Ecology, Vienna Veterinary University, Savoyenstraße 1, A-1160 Vienna, Austria (FS, AH), e-mail: franz.suchentrunk@vu-wien.ac.at; University of Forestry, Kl. Ochridski 10, Sofia (CM); Inst. of Zoology, Bulgarian Academy of Sciences, Tzar Osvoboditel 1, 1000 Sofia, Bulgaria (GM)

Key words: Lepus europaeus, allozymes, electrophoresis, genetic diversity, population genetics

\section{Introduction}

Brown hares Lepus europaeus (Pallas, 1778) from central Europe exhibit a moderate to somewhat elevated level of genetic variability, as compared to mammalian standards. The main portion of this variability is contained within local, rather than between geographical samples. No specific gene pools could be identified in 30 local samples from Austria, Poland and Hungary. Obviously, a fairly high level of gene flow among local populations results in a rather panmictic 
network, with little genetic differentiation over quite long geographical distances (Hartl et al. 1989, 1990, 1992, 1993, 1994).

The climate and vegetation of Europe during the last glaciation period (Lang 1994) suggest that brown hares were absent from most regions of Europe at that time. With the amelioration of the climate and the concomitant alteration of the late pleistocenic central European tundra vegetation into favourable habitats, brown hares likely started to colonize (recolonize?) central Europe from eastern and southeastern steppes and bushlands (and possibly also from southern Italy and southwestern Europe; cf eg Corbet 1986). On their way to central Europe they might have lost genetic variability (particularly in terms of numbers of alleles per locus), due to continued low effective population sizes of the invading and colonizing populations (cf eg, Nei et al. 1975, Hoffmann and Parsons 1997; see also Cwynar and MacDonald 1987 for lodgepole pine Pinus contorta in Western North America).

The present study focuses on the genetic variability contained in brown hare populations from Bulgaria. The southern and southeastern European refuge ranges of brown hares during the last glaciation period might have included this region. Genetic resources of brown hares might be richer in such a southeastern European source population maintained throughout the last glaciation period. This region was connected to Asia Minor by a landbridge during the late Pleistocene (eg, Lang 1994); hence, hares in this region might have received variant genes from Turkish populations. Gene flow may also have occurred (and still may occur) from Greece and from north and east of the Black sea. In fact, this southeastern European region can be considered as a zoogeographical crossroads, with a late pleistocenic connection to the particularly rich mammalian fauna of Asia Minor (cf, Cheylan 1991). The mammalian crossroad status of Southeast Europe is also indicated by the patterns of the present geographical distribution of several terrestrial mammalian species (eg, Microtus guentheri, M. majori, Apodemus mystacinus, Myomimus roachi, Cricetulus migratorius, Vormella peregusna, Canis aureus, cf Niethammer and Krapp 1978, 1982, Stubbe and Krapp 1993a, b). Increased genetic variability was detected in Bulgarian red deer Cervus elaphus and roe deer Capreolus capreolus as compared to central European populations (Hartl and Markov 1993). Alternatively, southwestern and southern European glacial refuges of brown hares cannot be entirely excluded. Pérez-Suárez et al. (1994) found great intraspecific nucleotide divergence in the mtDNA of brown hares from northern Spain, which might indicate two different immigration waves of brown hares to the Iberian Peninsula.

\section{Material and methods}

\section{Samples and enzyme assay}

Kidney and liver tissue samples of 157 hares were obtained in the course of a research program on heavy metal contamination (Tataruch et al. 1996) from eight sampling localities (SL) in Bulgaria 


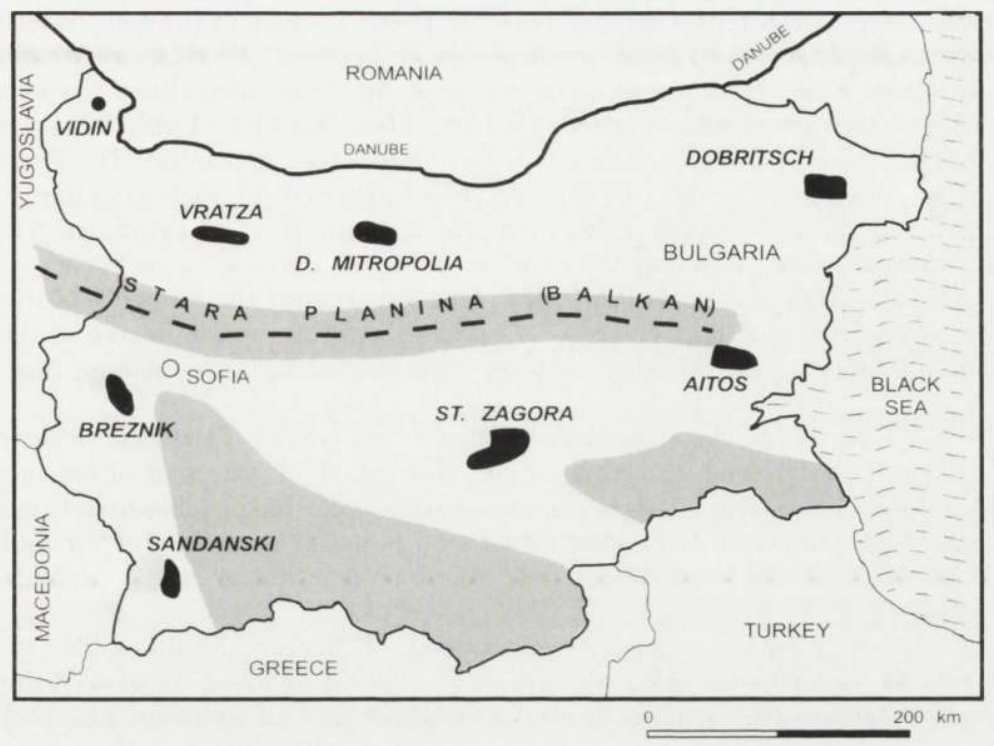

Fig. 1. Brown hare sampling localities (SLs) in Bulgaria (acronyms and sample sizes in parentheses): Breznik (Bre, $n=22$ ), D. Mitropolia (D.Mit, $n=20$ ), Vratza (Vra, $n=25$ ), Aitos (Ait, $n=20$ ), Sandanski (San, $n=19$ ), St. Zagora (St.Z, $n=15$ ), Vidin (Vid, $n=16$ ), Dobritsch (Dob, $n=20$ ). Stara Planina - main mountain range in east-west direction.

(Fig. 1). The hares were sexed by inspection of the internal reproductive organs and classified as young of the year or adults according to presence or absence of the lateral epiphysial protrusion of the ulna (cf, Suchentrunk et al. 1991).

Thirty-one isozymes/-systems encoded by 50 hypothetical structural gene loci (see Appendix) were assayed for allozymic variation by horizontal starch gel electrophoresis. The suite of loci studied is identical to the ones previously studied in Austrian brown hare populations (Hartl et al. 1993, Suchentrunk 1993, Hartl et al. 1994, 1995), except for Dia-1 (E.C. 1.6.2.2, for the corresponding isozyme, Acy-1 (E.C., 3.5.1.14), Ada-2, Ada-3 (E.C., 3.5.4.4), which have not been screened in the current study. The $\beta$-Gal locus was presently screened for allelic variability but excluded from further population genetic calculations because of the likelihood of occurrence of a null-allele, which could influence genotype frequencies (Hartl et al. 1993).

Tissue preparation, electrophoresis and protein specific staining followed Grillitsch et al. (1992). For resolving allelic variants direct side-by-side comparison of migrating allozymes was carried out including samples of the Austrian brown hares studied earlier (Hartl et al. 1993) on the same gels. For designation of alleles the nomenclature of Hartl et al. (1993) was used. The genotypes at polymorphic loci were determined in each specimen following the principles given in Rothe (1994). However, genotypes could not be determined for the entire set of loci in several individuals due to bad resolution producing ambiguous interpretations.

\section{Population genetic statistics}

The BIOSYS-1 pc package, release 1.7 (Swofford and Selander 1989) was used to calculate allele frequencies, average heterozygosity $\left(H_{o}\right.$ - observed, $H_{e}$ - expected), proportion of polymorphic loci $(P$, $99 \%$ crierion), mean number of alleles per locus based on all 49 loci $(A)$, deviation of observed genotypes at polymorphic loci from Hardy-Weinberg expectations, $F$-statistics for estimation of geograpical partitioning of genetic variability, Nei's (1978) unbiased genetic distances, modified 
Rogers' (cf Wright 1978) and Rogers' (1972) distances between all pairs of the eight Bulgarian SLs. To summarize genetic relationships in Bulgarian hares cluster analyses (UPGMA and Wagner algorithm, Farris 1972) were run, based on these distance matrices. All these calculations were carried out by including the enzyme data set produced in the same laboratory (see Hartl et al. 1993) of 469 Austrian brown hares, but adopting it to the presently studied 49 loci (see Appendix). The Shannon-Weaver information index $\left(H^{\prime}\right)$ based on allele frequencies was calculated for each sampling locality as an additonal estimator of genetic diversity, combining presence of alleles and associated frequencies (eg, Hedrick 1985, Hartl et al. 1994).

Wright's (1978) hierarchical $F$-statistics was employed to examine the influence of the two regions central Europe (= Austria) and southeastern Europe (= Bulgaria) on the degree of partitioning of genetic variability in relation to the much shorter geographical distances among SLs within each region.

Allele frequencies were tested for independence of age class (young of the year vs older animals) or sex by $\chi^{2}$-tests. Association of genotypes between loci was tested for each pair of polymorphic loci by the likelihood ratio for contingency tables of genotypes to check for linkage disequilibrium; significance of likelihood ratios were based on sequential Bonferroni procedures to account for multiple testing (Rice 1989). Bonferroni procedure was employed, whenever identical or partly dependent data sets were used in tests.

Variation of allele frequencies across the eight Bulgarian sampling localities (SL) were tested by $G$-tests, separately for each polymorphic locus. Variation of allele frequencies between the two regions southeastern (all Bulgarian SLs combined) and central Europe (all Austrian SLs combined) were tested by $\chi^{2}$-tests and Fisher's exact tests for cross tables with cells less than five alleles, and aggregated alleles in case of more than two alleles per locus. SL-specific values of $H_{o}, H_{e}, H^{\prime}, P$, and $A$ were tested for independence of sample size by Spearman rank correlations. Interrelationships between the diverse indices of SL-specific genetic variability were tested by one-tailed Spearman rank correlations. Average $H_{o}$-values were tested for significant variation across the Bulgarian SLs by a one-way ANOVA, based on arcsine-transformed individual heterozygosity values, and was tested variation of $P$ by a $G$-test. SL-specific $H_{o^{-}}, H_{e^{-}}, P-, A-$, and $H^{\prime}-$ values were tested for variation between Bulgaria and Austria by Kruskal-Wallis tests.

\section{Results}

Polymorphism was revealed at 11 loci of Bulgarian brown hares. There was no age or sex influence on genotype frequencies at any locus. Also, there was no significant association of genotypes between any pair of loci, indicating absence of linkage disequilibrium. Allele frequencies of polymorphic loci are listed in Table 1 for each Bulgarian sampling locality (SL), together with the range of respective allele frequencies found in the 20 Austrian samples. SL-specific indicators of genetic variability $\left(H_{o}, H_{e}, H^{\prime}, P, A\right)$ and significant deviation of genotypes from Hardy-Weinberg expectations are also given in Table 1. Frequencies of alleles varied significantly across the eight $(\mathrm{df}=7)$ Bulgarian SLs at the following loci: Idh-2 ( $p<0.001)$, Pgd $(p<0.001), H k-2(p<0.01), E s-1(p<0.001), E s-d(p<0.001)$, Pep-2 $(p<0.025)$, Mpi $(p<0.025)$. The overall allele frequencies (combined for all SLs per region) differed significantly between southeastern (Bulgaria) and central Europe (Austria) at the following loci: Mor-2 $(p=0.04), \operatorname{Idh}-2(p<0.0001), \operatorname{Pgd}$ $(p<0.0001), H k-2(p<0.0001), E s-1(p<0.0001), M p i(p<0.0001)$. None of the indices of SL-specific genetic variability did depend on sample size. Among the five indices of SL-specific genetic variability, a significant correlation was detected only 
Table 1. Allele frequencies at polymorphic loci of Bulgarian brown hares Lepus europaeus from eight sampling localities (for acronyms see Fig. 1) and respective ranges of allele frequencies of 20 Austrian sampling localities (20A). Deviations of observed genotype frequencies from Hardy-Weinberg expectations are indicated for respective loci and sampling localities at the right-hand side of the " 100 " allele by the associated probability value $\left({ }^{1}-p<0.01,{ }^{5}-p<0.05\right.$; exact probability). $n$ - number of individuals. $H_{o}$ - direct count heterozygosity, $H_{e}$ - expected heterozygosity, $H^{\prime}$ - Shannon-Weaver index of genetic diversity, $P$ - rate of polymorphism (99\% criterion), $A$ - mean number of alleles per locus.

\begin{tabular}{|c|c|c|c|c|c|c|c|c|c|c|}
\hline \multirow{2}{*}{ Locus } & \multirow{2}{*}{ Allele } & \multicolumn{8}{|c|}{ Bulgarian brown hare sampling localities } & \multirow{2}{*}{$\begin{array}{c}\begin{array}{c}\text { Austrian } \\
\text { samples }\end{array} \\
20 \mathrm{~A} \\
469\end{array}$} \\
\hline & & $\begin{array}{lc} & \text { Bre } \\
n & 22\end{array}$ & $\begin{array}{l}\text { D.Mit } \\
20\end{array}$ & $\begin{array}{l}\text { Vra } \\
25\end{array}$ & $\begin{array}{l}\text { Ait } \\
20\end{array}$ & $\begin{array}{c}\text { San } \\
19\end{array}$ & $\begin{array}{l}\text { St.Z } \\
15\end{array}$ & $\begin{array}{l}\text { Vid } \\
16\end{array}$ & $\begin{array}{c}\text { Dob } \\
20\end{array}$ & \\
\hline$S d h$ & $\begin{array}{l}100 \\
300\end{array}$ & $\begin{array}{l}1.0 \\
0.0\end{array}$ & $\begin{array}{l}1.0 \\
0.0\end{array}$ & $\begin{array}{l}1.0 \\
0.0\end{array}$ & $\begin{array}{l}1.0 \\
0.0\end{array}$ & $\begin{array}{l}1.0 \\
0.0\end{array}$ & $\begin{array}{l}1.0 \\
0.0\end{array}$ & $\begin{array}{l}1.0 \\
0.0\end{array}$ & $\begin{array}{l}0.975 \\
0.025\end{array}$ & $\begin{array}{l}0.933-1.0 \\
0.0-0.067\end{array}$ \\
\hline$L d h-2$ & $\begin{array}{r}100 \\
83\end{array}$ & $\begin{array}{l}1.0 \\
0.0\end{array}$ & $\begin{array}{l}1.0 \\
0.0\end{array}$ & $\begin{array}{l}1.0 \\
0.0\end{array}$ & $\begin{array}{l}1.0 \\
0.0\end{array}$ & $\begin{array}{l}1.0 \\
0.0\end{array}$ & $\begin{array}{l}1.0 \\
0.0\end{array}$ & $\begin{array}{l}0.969 \\
0.031\end{array}$ & $\begin{array}{l}1.0 \\
0.0\end{array}$ & $\begin{array}{l}0.955-1.0 \\
0.0-0.045\end{array}$ \\
\hline Mor-2 & $\begin{array}{r}100 \\
79\end{array}$ & $\begin{array}{l}1.0 \\
0.0\end{array}$ & $\begin{array}{l}1.0 \\
0.0\end{array}$ & $\begin{array}{l}1.0 \\
0.0\end{array}$ & $\begin{array}{l}1.0 \\
0.0\end{array}$ & $\begin{array}{l}1.0 \\
0.0\end{array}$ & $\begin{array}{l}1.0 \\
0.0\end{array}$ & $\begin{array}{l}1.0 \\
0.0\end{array}$ & $\begin{array}{l}1.0 \\
0.0\end{array}$ & $\begin{array}{l}0.833-1.0 \\
0.0-0.167\end{array}$ \\
\hline Idh-2 & $\begin{array}{r}100 \\
130 \\
83\end{array}$ & $\begin{array}{l}1.0 \\
0.0 \\
0.0\end{array}$ & $\begin{array}{l}0.55 \\
0.45 \\
0.0\end{array}$ & $\begin{array}{l}0.96^{1} \\
0.04 \\
0.0\end{array}$ & $\begin{array}{l}1.0 \\
0.0 \\
0.0\end{array}$ & $\begin{array}{l}0.5 \\
0.5 \\
0.0\end{array}$ & $\begin{array}{l}0.867 \\
0.133 \\
0.0\end{array}$ & $\begin{array}{l}0.969 \\
0.031 \\
0.0\end{array}$ & $\begin{array}{l}1.0 \\
0.0 \\
0.0\end{array}$ & $\begin{array}{l}1.0-0.732 \\
0.0-0.232 \\
0.0-0.045\end{array}$ \\
\hline Pgd & $\begin{array}{r}100 \\
170 \\
129 \\
117 \\
64\end{array}$ & $\begin{array}{l}0.795 \\
0.0 \\
0.046 \\
0.159 \\
0.0\end{array}$ & $\begin{array}{l}0.711^{1} \\
0.0 \\
0.0 \\
0.263 \\
0.026\end{array}$ & $\begin{array}{l}0.587^{5} \\
0.0 \\
0.065 \\
0.283 \\
0.065\end{array}$ & $\begin{array}{l}0.75 \\
0.0 \\
0.036 \\
0.143 \\
0.071\end{array}$ & $\begin{array}{l}0.456 \\
0.0 \\
0.273 \\
0.273 \\
0.0\end{array}$ & $\begin{array}{l}0.766 \\
0.0 \\
0.0 \\
0.167 \\
0.067\end{array}$ & $\begin{array}{l}0.531 \\
0.0 \\
0.0 \\
0.438 \\
0.031\end{array}$ & $\begin{array}{l}0.9 \\
0.0 \\
0.0 \\
0.1 \\
0.0\end{array}$ & $\begin{array}{l}0.825-1.0 \\
0.0-0.071 \\
0.0-0.083 \\
0.0-0.037 \\
0.0-0.143\end{array}$ \\
\hline$H k-2$ & $\begin{array}{r}100 \\
67\end{array}$ & $\begin{array}{l}0.977 \\
0.023\end{array}$ & $\begin{array}{l}0.974 \\
0.026\end{array}$ & $\begin{array}{l}1.0 \\
0.0\end{array}$ & $\begin{array}{l}1.0 \\
0.0\end{array}$ & $\begin{array}{l}1.0 \\
0.0\end{array}$ & $\begin{array}{l}1.0 \\
0.0\end{array}$ & $\begin{array}{l}1.0 \\
0.0\end{array}$ & & $\begin{array}{l}0.952-1.0 \\
0.0-0.048\end{array}$ \\
\hline Es-1 & $\begin{array}{l}-100 \\
-108 \\
-75 \\
-42\end{array}$ & $\begin{array}{l}0.45 \\
0.0 \\
0.5 \\
0.05\end{array}$ & $\begin{array}{l}0.211 \\
0.079 \\
0.658 \\
0.052\end{array}$ & $\begin{array}{l}0.435 \\
0.0 \\
0.522 \\
0.043\end{array}$ & $\begin{array}{l}0.083 \\
0.028 \\
0.75 \\
0.139\end{array}$ & $\begin{array}{l}0.176 \\
0.029 \\
0.795 \\
0.0\end{array}$ & $\begin{array}{l}0.2 \\
0.0 \\
0.667 \\
0.133\end{array}$ & $\begin{array}{l}0.281 \\
0.0 \\
0.719 \\
0.0\end{array}$ & $\begin{array}{l}0.425 \\
0.0 \\
0.45 \\
0.125\end{array}$ & $\begin{array}{l}0.357-0.833 \\
0.0-0.143 \\
0.167-0.550 \\
0.0-0.017\end{array}$ \\
\hline$E s-d$ & $\begin{array}{l}100 \\
141\end{array}$ & $\begin{array}{l}1.0 \\
0.0\end{array}$ & $\begin{array}{l}0.8 \\
0.2\end{array}$ & $\begin{array}{l}0.826 \\
0.174\end{array}$ & $\begin{array}{l}0.95 \\
0.05\end{array}$ & $\begin{array}{l}1.0 \\
0.0\end{array}$ & $\begin{array}{l}0.867 \\
0.133\end{array}$ & $\begin{array}{l}0.781 \\
0.219\end{array}$ & $\begin{array}{l}0.8 \\
0.2\end{array}$ & $\begin{array}{l}0.672-0.983 \\
0.017-0.328\end{array}$ \\
\hline$\beta-G a l$ & $\begin{array}{r}100 \\
117 \\
83\end{array}$ & $\begin{array}{l}0.75^{1} \\
0.0 \\
0.25\end{array}$ & $\begin{array}{l}0.842 \\
0.132 \\
0.026\end{array}$ & $\begin{array}{l}0.35^{1} \\
0.125 \\
0.525\end{array}$ & $\begin{array}{l}0.722 \\
0.167 \\
0.111\end{array}$ & $\begin{array}{l}0.75^{5} \\
0.222 \\
0.028\end{array}$ & $\begin{array}{l}0.733 \\
0.1 \\
0.167\end{array}$ & $\begin{array}{l}0.688^{1} \\
0.156 \\
0.156\end{array}$ & $\begin{array}{l}0.708 \\
0.167 \\
0.125\end{array}$ & $\begin{array}{l}0.125-0.810 \\
0.0-0.250 \\
0.0-0.875\end{array}$ \\
\hline Pep-2 & $\begin{array}{r}100 \\
104 \\
94\end{array}$ & $\begin{array}{l}0.818 \\
0.182 \\
0.0\end{array}$ & $\begin{array}{l}0.725 \\
0.275 \\
0.0\end{array}$ & $\begin{array}{l}0.826 \\
0.174 \\
0.0\end{array}$ & $\begin{array}{l}0.842 \\
0.158 \\
0.0\end{array}$ & $\begin{array}{l}0.75 \\
0.194 \\
0.056\end{array}$ & $\begin{array}{l}0.933 \\
0.067 \\
0.0\end{array}$ & $\begin{array}{l}0.938 \\
0.062 \\
0.0\end{array}$ & $\begin{array}{l}0.825^{1} \\
0.175 \\
0.0\end{array}$ & $\begin{array}{l}0.618-0.900 \\
0.075-0.382 \\
0.0\end{array}$ \\
\hline Mpi & $\begin{array}{r}100 \\
126 \\
77\end{array}$ & $\begin{array}{l}0.977 \\
0.023 \\
0.0\end{array}$ & $\begin{array}{l}0.975 \\
0.025 \\
0.0\end{array}$ & $\begin{array}{l}0.935 \\
0.065 \\
0.0\end{array}$ & $\begin{array}{l}0.95 \\
0.05 \\
0.0\end{array}$ & $\begin{array}{l}0.806 \\
0.194 \\
0.0\end{array}$ & $\begin{array}{l}0.967 \\
0.033 \\
0.0\end{array}$ & $\begin{array}{l}0.875 \\
0.125 \\
0.0\end{array}$ & $\begin{array}{l}0.875 \\
0.1 \\
0.025\end{array}$ & $\begin{array}{l}0.914-1.0 \\
0.0-0.086 \\
0.0-0.067\end{array}$ \\
\hline$H_{o}$ & & 0.017 & 0.036 & 0.033 & 0.025 & 0.042 & 0.026 & 0.029 & 0.031 & $0.020-0.039$ \\
\hline$H_{e}$ & & 0.026 & 0.046 & 0.038 & 0.026 & 0.045 & 0.031 & 0.035 & 0.034 & $0.022-0.033$ \\
\hline$H^{\prime}$ & & 2.16 & 3.03 & 3.2 & 2.43 & 3.54 & 2.72 & 2.67 & 2.71 & $1.70-2.73$ \\
\hline$P$ & & 10.2 & 14.3 & 12.2 & 10.2 & 10.2 & 12.2 & 14.3 & 12.2 & $8.2-16.3$ \\
\hline & & 1.14 & 1.2 & 1.18 & 1.18 & 1.16 & 1.16 & 1.16 & 1.16 & $1.08-1.20$ \\
\hline
\end{tabular}


Table 2. Comparison of genetic variability in Bulgarian and Austrian sampling localities. Mean, standard deviation (SD) and range are given of the following indices: $H_{o}, H_{e}, H^{\prime}, P, A$ - for explanation of indices see Table 1, $n$ - number of local samples, $p$ - significance level (threshold value for multiple testing at a nominal $\alpha=0.05$ according to Boferroni procedure is 0.01 ). ns - not significant.

\begin{tabular}{|c|c|c|c|c|c|c|c|}
\hline \multirow{2}{*}{ Index } & \multicolumn{3}{|c|}{$\begin{array}{l}\text { Bulgaria } \\
\text { (southeastern Europe), } n=8\end{array}$} & \multicolumn{3}{|c|}{$\begin{array}{c}\text { Austria } \\
\text { (central Europe), } n=20\end{array}$} & \multirow[b]{2}{*}{$p$} \\
\hline & Mean & SD & Range & Mean & SD & Range & \\
\hline$H_{o}$ & 0.03 & 0.007 & $0.017-0.042$ & 0.03 & 0.005 & $0.02-0.039$ & ns \\
\hline$H_{e}$ & 0.035 & 0.007 & $0.026-0.045$ & 0.027 & 0.003 & $0.022-0.033$ & $<0.0001$ \\
\hline$H^{\prime}$ & 2.7 & 0.4 & $2.1-3.7$ & 2.2 & 0.3 & $1.7-2.7$ & 0.0037 \\
\hline$P$ & 12.0 & 1.6 & $10.2-14.3$ & 10.7 & 2.0 & $8.2-16.3$ & $\mathrm{~ns}$ \\
\hline$A$ & 1.17 & 0.02 & $1.14-1.2$ & 1.13 & 0.03 & $1.08-1.2$ & ns \\
\hline
\end{tabular}

Table 3. Locus-specific expected heterozygosities in \% ( $\beta$-Gal not considered). Mean value and range are given for Bulgarian and Austrian sampling localities (SLs). For Austrian SLs see Hartl et al. (1993). p-significance values for differences between Bulgarian and Austrian SLs (threshold value for significance is 0.005 following a Bonferroni procedure with a nominal $\alpha=0.05$ ).

\begin{tabular}{|c|c|c|c|c|c|}
\hline \multirow{2}{*}{ Locus } & \multicolumn{2}{|c|}{ Bulgarian SLs } & \multicolumn{2}{|c|}{ Austrian SLs } & \multirow[b]{2}{*}{$p$} \\
\hline & Mean value & Range & Mean value & Range & \\
\hline$S d h$ & 0.6 & $0.0-4.9$ & 0.6 & $0.0-12.4$ & $\mathrm{~ns}$ \\
\hline$L d h-2$ & 0.8 & $0.0-6.0$ & 0.4 & $0.0-8.7$ & $\mathrm{~ns}$ \\
\hline Mor-2 & 0.0 & - & 3.6 & $0.0-27.8$ & ns \\
\hline$I d h-2$ & 17.1 & $0.0-50.0$ & 6.3 & $0.0-40.9$ & $\mathrm{~ns}$ \\
\hline$P g d$ & 43.4 & $18.0-64.5$ & 12.0 & $0.0-36.0$ & $<0.0001$ \\
\hline$H k-2$ & 1.2 & $0.0-5.1$ & 1.5 & $0.0-9.5$ & ns \\
\hline Es-1 & 48.1 & $33.7-60.1$ & 48.0 & $27.8-63.0$ & $\mathrm{~ns}$ \\
\hline$E s-d$ & 15.7 & $0.0-32.0$ & 27.9 & $3.4-44.1$ & $\mathrm{~ns}$ \\
\hline Pep-2 & 27.2 & $11.7-27.2$ & 30.4 & $13.9-30.4$ & ns \\
\hline$M p i$ & 14.13 & $4.4-31.3$ & 3.9 & $0.0-36.0$ & 0.003 \\
\hline
\end{tabular}

Table 4. Geographical partitioning of genetic variability in Bulgarian brown hares. Pairwise $F_{S T}$ values between sampling localities above, and associated significance level $(p)$ below diagonal $(*-0.001<$ $p<0.005,{ }^{* *}-p<0.001, \mathrm{~ns}-$ not significantly different from zero). For sampling localities see Fig. 1.

\begin{tabular}{lcccccccc}
\hline & $(1)$ & $(2)$ & $(3)$ & $(4)$ & $(5)$ & $(6)$ & $(7)$ & $(8)$ \\
\hline (1) Breznik & - & 0.08 & 0.02 & 0.04 & 0.12 & 0.04 & 0.06 & 0.02 \\
(2) D. Mitropolia & $* *$ & - & 0.05 & 0.07 & 0.04 & 0.04 & 0.06 & 0.07 \\
(3) Vratza & $*$ & $* *$ & - & 0.04 & 0.08 & 0.03 & 0.02 & 0.02 \\
(4) Aitos & $* *$ & $* *$ & $* *$ & - & 0.10 & 0.02 & 0.05 & 0.05 \\
(5) Sandanski & $* *$ & $*$ & $* *$ & $* *$ & - & 0.08 & 0.09 & 0.13 \\
(6) St. Zagora & $\mathrm{ns}$ & $*$ & $\mathrm{~ns}$ & $\mathrm{~ns}$ & $*$ & - & 0.03 & 0.037 \\
(7) Vidin & $*$ & $* *$ & $\mathrm{~ns}$ & $\mathrm{~ns}$ & $* *$ & $\mathrm{~ns}$ & - & 0.06 \\
(8) Dobritsch & $*$ & $* *$ & $\mathrm{~ns}$ & $* *$ & $* *$ & $\mathrm{~ns}$ & $*$ & - \\
\hline
\end{tabular}


Table 5. Wright's (1978) hierarchical $F$-statistics indicating the degree of partitioning of relative genetic variability in brown hares due to geographical distribution within and between the two regions southeastern Europe (Bulgaria) and central Europe (Austria) as compared to partitioning due to sampling localities irrespective of the region. Values calculated over all polymorphic loci.

\begin{tabular}{lcc}
\hline Comparison $(\mathrm{X}-\mathrm{Y})$ & Variance component & $F_{\mathrm{XY}}$ \\
\hline Sampling locality - region & 0.0715 & 0.046 \\
Region - total sample & 0.0580 & 0.036 \\
Sampling locality - total sample & 0.1295 & 0.080 \\
\hline
\end{tabular}

between $H_{o}$ and $H_{e}(p<0.0001)$. Hence, all other indices provided additional information on genetic variability. $H_{o}$-values varied significantly $(p=0.0049)$ across Bulgarian SLs but $P$-values did not. The statistics of the comparison of the indices of SL-specific genetic variability of hares from Bulgaria and Austria is presented in Table 2. Significant differences of locus-specific expected heterozygosities between the SLs from Bulgaria and Austria (Table 3 ) were found only at two loci: Pgd ( $p=0.0028), M p i(p=0.0001)$.

Table 6. Genetic distances among the eight Bulgarian and the 20 Austrian brown hare sampling localities. Nei's (1978) genetic distance above and Rogers' modified distances below the diagonal. (1)-(8): Bulgarian sampling localities. Ranges of pairwise genetic distances (Nei's $1978 \mathrm{D}$ above and Rogers' modified distances below values) between Bulgarian and 20 Austrian sampling localities (cf Hartl et al. 1993) are given in the right section of the table.

\begin{tabular}{|c|c|c|c|c|c|c|c|c|c|}
\hline & (1) & (2) & (3) & (4) & (5) & (6) & (7) & (8) & $\begin{array}{l}\text { Distance range } \\
\text { with } 20 \\
\text { Austrian local } \\
\text { samples }\end{array}$ \\
\hline (1) Breznik & - & 0.005 & 0.001 & 0.002 & 0.009 & 0.001 & 0.003 & 0.001 & $\begin{array}{l}0.000-0.004 \\
0.029-0.069\end{array}$ \\
\hline (2) D. Mitropolia & 0.079 & - & 0.004 & 0.005 & 0.002 & 0.002 & 0.005 & 0.005 & $\begin{array}{l}0.005-0.011 \\
0.078-0.106\end{array}$ \\
\hline (3) Vraza & 0.037 & 0.068 & - & 0.002 & 0.007 & 0.001 & 0.000 & 0.001 & $\begin{array}{l}0.001-0.005 \\
0.026-0.076\end{array}$ \\
\hline (4) Aitos & 0.048 & 0.074 & 0.052 & - & 0.007 & 0.000 & 0.002 & 0.002 & $\begin{array}{l}0.001-0.011 \\
0.023-0.107\end{array}$ \\
\hline (5) Sandanski & 0.097 & 0.057 & 0.087 & 0.088 & - & 0.005 & 0.006 & 0.011 & $\begin{array}{l}0.010-0.019 \\
0.105-0.137\end{array}$ \\
\hline (6) St. Zagora & 0.046 & 0.058 & 0.043 & 0.030 & 0.080 & - & 0.001 & 0.001 & $\begin{array}{l}0.001-0.008 \\
0.030-0.097\end{array}$ \\
\hline (7) Vidin & 0.062 & 0.075 & 0.037 & 0.054 & 0.086 & 0.046 & - & 0.003 & $\begin{array}{l}0.003-0.011 \\
0.067-0.106\end{array}$ \\
\hline (8) Dobritsch & 0.036 & 0.079 & 0.041 & 0.055 & 0.106 & 0.046 & 0.063 & - & $\begin{array}{l}0.000-0.003 \\
0.023-0.059\end{array}$ \\
\hline
\end{tabular}




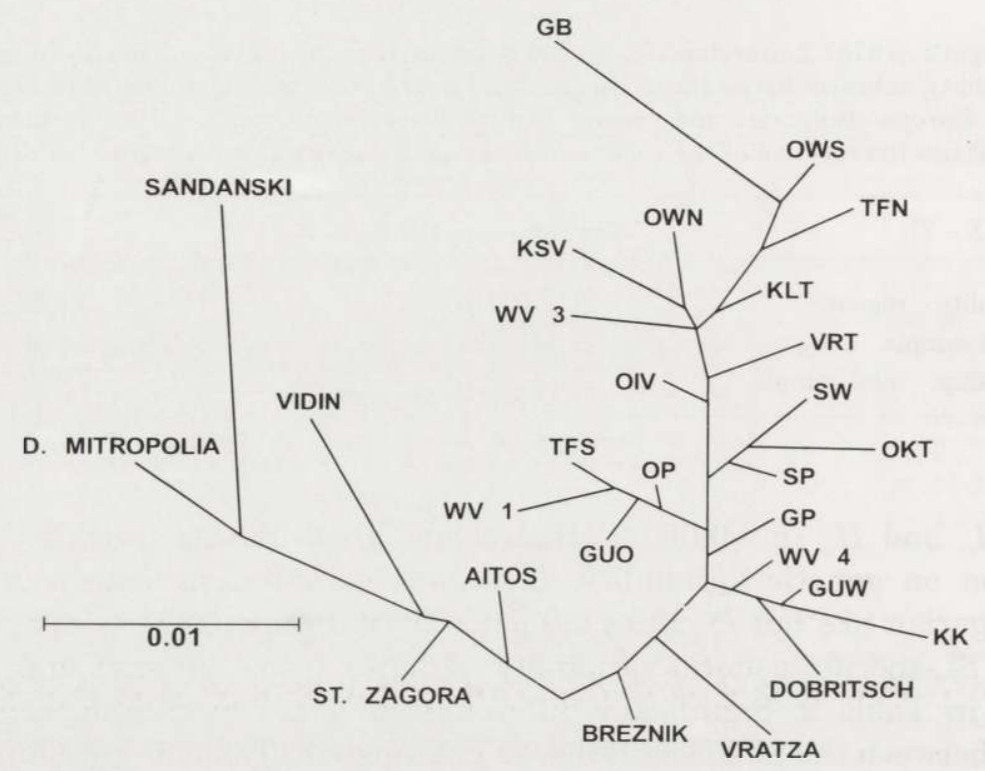

Fig. 2. Unrooted Wagner dendrogram based on Rogers' (1972) distances depicting genetic relationships among the eight Bulgarian and the 20 Austrian local brown hare populations. For the acronyms of the Austrian local populations see Hartl et al. (1993). Cophenetic correlation coefficient $=0.931$, distance between Mitropolia and Sandanski $=0.018$.

The level of relative genetic differentiation $\left(F_{S T}\right.$ values, Table 4$)$ between the Bulgarian SLs was generally low, but nevertheless, many pairwise values differed significantly from zero, as inferred from comparisons of allele frequencies. Wright's (1978) hierarchical $F$-statistics (Table 5) revealed that partitioning of relative genetic variability over long geographic distances [southeastern Europe (= Bulgaria) vs central Europe (=Austria)] was roughly of the same low magnitude as among SLs within the regions (Bulgaria and Austria, respectively).

The matrices of genetic distances (Nei's $1978 \mathrm{D}$, and modified Rogers' distances) between Bulgarian SLs and the ranges of pairwise distances with the re-calculated Austrian SLs are given in Table 6. The genetic relationships of brown hares from the eight Bulgarian SLs and the 20 Austrian SLs are summarized by an unrooted Wagner dendrogram based on Rogers' (1972) distances in Fig. 2. The rooted UPGMA cluster yielded essentially the same pattern of differentiation.

\section{Discussion}

The present study revealed only a slightly increased level of allozymic diversity in brown hares from Bulgaria as compared to large areas of central Europe. This is indicated by the significant differences of expected heterozygosity $\left(H_{e}\right)$ and Shannnon Weaver diversity $\left(H^{\prime}\right)$. However, SL-specific rates of polymorphism and 
average numbers of alleles per locus $(A)$ do not differ significantly between Bulgaria and central Europe (Austria). The overall rate of polymorphism (99\% criterion; calculated over all specimens combined from the SLs from Bulgaria and Austria, respectively) amounts to $22 \%$ in Bulgaria and to $24 \%$ in Austria, with inclusion of the $\beta$-Gal locus. The marginally but significantly increased level of genetic diversity in the Bulgarian hares is basically due to increased heterozygosities at the Pgd and Mpi loci (Table 3).

The lack of a raised rate of polymorphism in Bulgaria is due to the absence of any new polymorphic locus as compared to central Europe, and the absence of polymorphism at the Mor-2 locus in Bulgarian hares. This locus exhibits two alleles $\left(\right.$ Mor- $2^{100}$, Mor- $\left.2^{79}\right)$ in some local populations from western and southern Austria anc from one locality in Hungary, with generally low frequencies of the Mor- $2^{79}$ allele (Hartl et al. 1990, 1993, 1994). In Poland, the Mor- ${ }^{79}$ allele is obviously disiributed over large areas, but also only at low frequencies (Hartl et al. 1992). According to unpublished data (F. Suchentrunk, in prep.), this allele is present in several other local populations of northwestern continental Europe, too. The absence of this allele in Bulgarian brown hares, opposed to its widespread occurrence in continental Europe, may indicate an origin outside southeastern Europe. In addition, several other alleles $\left(I d h-2^{83}, P g d^{170}, P e p-2^{114}\right)$, that have been found in some of the central European populations, could not be detected in the Bugarian brown hares, despite a fairly large sample size. This fact, in conjunction with the above reported pattern of distribution of the Mor- $2^{79}$, is in accordance with the hypothesis that central European gene pools of brown hares may have been exposed to gene flow from regions outside of southeastern Europe. It is not likely that alleles at several loci have evolved within the short time of (re-)colonization of certral Europe. Even if evolution of these alleles in central European populations is assumed, one might expect that these alleles would be distributed more locally, and not over a wide range, as is the case for the rare alleles Mor- $2^{79}$ and $P g d^{170}$.

The presently studied Bulgarian hares harbour only one allele $\left(P e p^{94}\right)$, which has not yet been found in any of the samples of central European brown hares. This allele occurs at a low frequency in only one of the Bulgarian SLs (Sandanski, Fig.1), which is situated in the Struma Valley of southwestern Bulgaria. The Struma valey connects northern Greece with this part of Bulgaria. Hence this genetic variant might indicate limited gene flow from northern Greece to Bulgaria. However, the absence of any other new allele in Bulgarian hares suggests either the lacs of a strong gene flow from Asia Minor over the late-glacial landbridge and from other areas (Greece, northeast of the Black Sea), or the absence of other common alleles in such possible source populations.

Generally, a low level of partitioning of relative genetic variability is indicated by the pairwise $F_{S T}$ values among the geograpahical samples in Bulgaria. The Stara Planina mountain range (Balkan) apparently has no influence on the distribution of senetic variants. The low level of geographic partitioning of the gene pool of Bugarian hares conforms to the little genetic differentiation found already in 
central European brown hares; it might be partly due to intensive transfers of hares (500-1000 hares, two to three times per year between 1970 and 1980, particularly from eastern to western regions in Bulgaria). The hierarchical $F$-statistics demonstrates an only small effect of long geographical distances on the distribution of genetic diversity between central and southeastern Europe: less than four percent of the relative genetic diversity was partitioned between central and southeastern Europe, and about the same amount of genetic diversity was due to partitioning among local samples within the two regions. Accordingly, little absolute genetic differentiation occurred among regional and inter-regional samples from Bulgaria and central Europe (Table 6; cf also Hartl et al. 1990, 1992, 1993). This implies considerable gene flow across long geographical distances leading to a rather panmictic network of local populations; it also suggests that the absence of new common alleles in the Bulgarian brown hares is due to the lack of such new common alleles in various southern and southeastern parts of the distributional range of brown hares. Despite the general picture of a high level of gene flow over long geographic distances, there is a tendency of a shallow gene pool divergence between local populations of southeastern (Bulgarian) and central (Austrian) European brown hares (Fig. 2). This might be partly produced by gene flow to central Europe from other regions than southeastern Europe (see Taberlet et al. 1998 for a portrayal of immigration routes of plants and animals into central Europe). Further studies of brown hare populations from northwestern, southwestern and southern European regions, together with mtDNA RFLPs (as indicated by the study of Pérez-Suárez et al. 1994) could provide tests for the hypothesis that different glacial refuges might have functioned as source populations for gene flow to the extant gene pool of central European brown hares.

Acknowledgements: We would like to thank Prof Frieda Tataruch (Res. Inst. of Wildlife Ecology, Vienna) for providing the tissue samples that were collected in the course of an other project, and Mr A. Körber (Res. Inst. of Wildl. Ecol., Vienna) for his assistence with the graphic work. Suggestions of three anonymous referees improved the manuscript.

\section{References}

Cheylan G. 1991. Patterns of Pleistocene turnover, current distribution and speciation among Mediterranean mammals. [In: Biogeography of Mediterranean Invasions, R. H. Groves, F. Di Castri, eds]. Cambridge University Press, Cambridge: 227-262.

Corbet G. B. 1986. Relationships and origins of the European lagomorphs. Mammal Review 16: $105-110$.

Cwynar L. C. and MacDonald G. M. 1987. Geographical variation of lodgepole pine in relation to population history. The American Naturalist 129: 463-469.

Farris J. S. 1972. Estimating phylogenetic trees from distance matrices. The American Naturalist 106: 645-668.

Grillitsch M., Hartl G. B., Suchentrunk F. and Willing R. 1992. Allozyme evolution and the molecular clock in the Lagomorpha. Acta Theriologica 37: 1-13.

Hartl G. B. and Markov G. 1993. Genetic diversity in Bulgarian deer species. Comptes rendus de i'Académie bulgare des Sciences 46: 89-92. 
Hartl G. B., Markowski J., Kovacs G., Grillitsch M. and Willing R. 1990. Biochemical variation and differentiation in the brown hare (Lepus europaeus) of Central Europe. Zeitschrift für Säugetierkunde 55: 186-193.

Hartl G. B., Markowski J., Świątecki A., Janiszewski T. and Willing R. 1992. Genetic diversity in the Polish brown hare Lepus europaeus Pallas, 1778: implications for conservation and management. Acta Theriologica 37: 15-25

Hartl G. B., Suchentrunk F., Nadlinger K. and Willing R. 1993. An integrative analysis of genetic differentiation in the brown hare Lepus europaeus based on morphology, allozymes, and mitochondrial DNA. Acta Theriologica 38, Suppl. 2: 33-57.

Hartl G. B., Suchentrunk F., Willing R. and Grillitsch M. 1989. Biochemisch-genetische Variabilität und Differenzierung beim Feldhasen (Lepus europaeus) in Niederösterreich. Wiener Tierärztliche Monatsschrift 76: 279-284.

Hartl G. B., Suchentrunk F., Willing R. and Petznek R. 1995. Allozyme heterozygosity and fluctuating asymmetry in the brown hare (Lepus europaeus): a test of the developmental homeostasis hypothesis. Philosophical Transactions of the Royal Society London B 350: 313-323.

Hartl G. B., Willing R. and Nadlinger K. 1994. Allozymes in mammalian population genetics and systematics: Indicative function of a marker system reconsidered. [In: Molecular ecology and evolution: Approaches and Applications. B. Schierwater, B. Streit, G. P. Wagner and R. DeSalle, eds]. Birkhäuser Verlag, Basel, Switzerland: 299-310.

Hedrick P. W. 1985. Genetics of Populations. Jones \& Bartlett Publ., Inc. Boston: 1-629.

Hoffmann A. A. and Parsons P. A. 1997. Extreme Environmental Change and Evolution. Cambridge University Press, Cambridge: 1-259.

Lang G. 1994. Quartäre Vegetationsgeschichte Europas. Methoden und Ergebnisse. G. Fischer, Jena: $1-462$.

Nei M., Maruyama T. and Chakraborty R. 1975. The bottleneck effect and genetic variability in populations. Evolution 29: 1-10.

Nei M. 1978. Estimation of average heterozygosity and genetic distance from a small number of individuals. Genetics 89: 583-590.

Niethammer J. and Krapp F. (eds) 1978. Handbuch der Säugetiere Europas. Vol. 1/Nagetiere 1. Akademische Verlagsgesellschaft Wiesbaden: 1-476.

Niethammer J. and Krapp F. (eds) 1982. Handbuch der Säugetiere Europas. Vol. 2/Nagetiere II. Akademische Verlagsgesellschaft Wiesbaden: 1-649.

Pérez-Suárez G., Palacios F. and Boursot P. 1994. Speciation and paraphyly in western mediterranean hares (Lepus castroviejoi, L. europaeus, L. granatensis, and L. capensis) revealed by mitochondrial DNA phylogeny. Biochemical Genetics 32: 423-436.

Rice W. S. 1989. Analyzing tables of statistical tests. Evolution 43: 223-225.

Rogers J. S. 1972. Measures of genetic similarity and genetic distance. Studies in Genetics. University of Texas Publications 7213: 145-153.

Rothe G. M. 1994. Electrophoresis of enzymes. Laboratory Methods. Springer LAB Manual. Springer Verlag, Berlin: 1-307.

Stubbe M. and Krapp F. 1993a. Handbuch der Säugetiere Europas. Raubsäuger Teil I. Aula Verlag, Wiesbaden: 1-526.

Stubbe M. and Krapp F. 1993b. Handbuch der Säugetiere Europas. Raubsäuger Teil I. Aula Verlag, Wiesbaden: 529-1213.

Suchentrunk F. 1993. Variability of minor tooth traits and allozymic diversity in brown hare Lepus europaeus populations. Acta Theriologica 38, Suppl. 2: 59-69.

Suchentrunk F., Willing R. and Hartl G. B. 1991. On eye lens weights and other age criteria of the Brown hare (Lepus europaeus Pallas, 1778). Zeitschrift für Säugetierkunde 56: 365-374.

Swofford D. L. and Selander R. B. 1989. BIOSYS-1. A computer program for the analysis of allelic variation in population genetics and biochemical systematics. Release 1.7. Users manual. Illinois Natural History Survey, Champaign. 
Taberlet P., Fumagalli L., Wust-Saucy A. and Cosson J. 1998. Comparative phylogeography and postglacial colonization routes in Europe. Molecular Ecology 7: 453-464.

Tatatruch F., Onderscheka K., Botev N., Ninov N. and Mihailov C. 1996. Die Schwermetallbelastung der freilebenden Wildtiere in Bulgarien. [In: Proc. XII IUGB Congr. "The Game and the Man", Sofia, Bulgaria, Sept. 1995., N. Botev, S. Golovatch and N. Penev, eds]: 432-438.

Wright S. 1978. Evolution and genetics of populations. Vol. IV. Variability within and among natural populations. University Chicago Press, Chicago: 1-580.

Received 26 October 1998, accepted 15 April 1999.

Appendix. List of enzyme loci assayed for allozymic variation.

\begin{tabular}{|c|c|c|c|}
\hline Isozyme/-system & Abbreviation & E.C. number & Locus \\
\hline$\alpha$-glycerophosphate dehydrogenase & GDC & 1.1.1.8 & $G d c$ \\
\hline Sorbitol dehydrogenase & $\mathrm{SDH}$ & 1.1.1.14 & $S d h$ \\
\hline Lactate dehydrogenase & $\mathrm{LDH}$ & 1.1.1.27 & $L d h-1,-2$ \\
\hline Malate dehydrogenase & MOR & 1.1.1.37 & Mor $-1,-2$ \\
\hline Malic enzyme & MOD & 1.1 .1 .40 & Mod-1, -2 \\
\hline Isocitrate dehydrogenase & IDH & 1.1.1.42 & $I d h-1,-2$ \\
\hline 6-phosphogluconate dehydrogenase & PGD & 1.1.1.44 & Pgd \\
\hline Glucose dehydrogenase & GDH & 1.1.1.47 & $G d h-2$ \\
\hline Glucose-6-phosphate dehydrogenase & GPD & 1.1.1.49 & Gpd \\
\hline Glyceraldehyde-3-phosphate dehydrogenase & GAPDH & 1.2 .1 .12 & Gapdh \\
\hline Xanthine dehydrogenase & $\mathrm{XDH}$ & 1.2.3.2 & $X d h$ \\
\hline Glutamate dehydrogenase & GLUD & 1.4.1.3 & Glud \\
\hline Catalase & CAT & 1.11.1.6 & Cat \\
\hline Superoxide dismutase & SOD & 1.15.1.1 & Sod $-1,-2$ \\
\hline Purine nucleoside phosphorylase & NP & 2.4 .2 .1 & $N p$ \\
\hline Aspartate aminotransferase & AAT & 2.6.1.1 & Aat $-1,-2$ \\
\hline Hexokinase & HK & 2.7.1.1 & $H k-1,-2,-3$ \\
\hline Pyruvate kinase & PK & 2.7.1.40 & $P k-1$ \\
\hline Creatine kinase & CK & 2.7.3.2 & $C k-1,-2$ \\
\hline Adenylate kinase & $\mathrm{AK}$ & 2.7.4.3 & $A k-1,-2$ \\
\hline Phosphoglucomutase & PGM & 2.7.5.1 & Pgm-1,-2,-3 \\
\hline Esterases & ES & 3.1.1.1 & Es-1; 4.2.1.1, Es-d \\
\hline Acid phosphatase & $\mathrm{ACP}$ & 3.1.3.2 & Acp-1,-2,-3 \\
\hline Fructose-1,6-diphosphatase & FDP & 3.1.3.11 & $F d p$ \\
\hline B-galactosidase & B-GAL & 3.2 .1 .23 & $\beta-G a l$ \\
\hline Peptidases & PEP & 3.4 .11 & Pep-1,-2 \\
\hline Guanine deaminase & GDA & 3.5 .4 .3 & Gda \\
\hline Aldolase & ALDO & 4.1.2.13 & Aldo \\
\hline Fumarate hydratase & $\mathrm{FH}$ & 4.2.1.2 & $F h$ \\
\hline Aconitase & $\mathrm{ACO}$ & 4.2.1.3 & Aco- $1,-2$ \\
\hline Mannose phosphate isomerase & MPI & 5.3.1.8 & $M p i$ \\
\hline Glucose phosphate isomerase & GPI & 5.3 .1 .9 & Gpi-1,-2 \\
\hline
\end{tabular}

